\title{
Image Reconstruction of Imatron Electron-Beam Tomography Data
}

\author{
Kyle Champley, Steve Azevedo, Dan Schneberk, Harry Martz \\ Lawrence Livermore National Laboratory \\ Livermore, CA 94551
}

Work performed on the

Science \& Technology Directorate of the

Department of Homeland Security

Statement of Work

June 22, 2012

(IM \# 631272)

LLNL-TR-577692

Version \#1
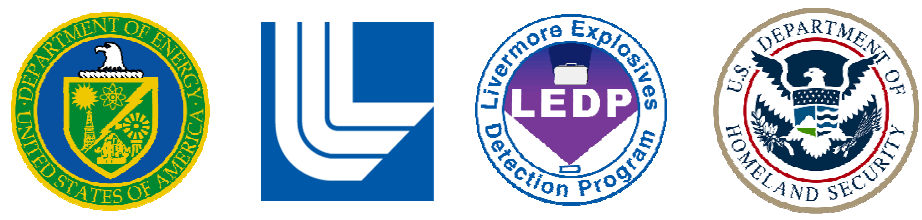
This document was prepared as an account of work sponsored by an agency of the United States government. Neither the United States government nor Lawrence Livermore National Security, LLC, nor any of their employees makes any warranty, expressed or implied, or assumes any legal liability or responsibility for the accuracy, completeness, or usefulness of any information, apparatus, product, or process disclosed, or represents that its use would not infringe privately owned rights. Reference herein to any specific commercial product, process, or service by trade name, trademark, manufacturer, or otherwise does not necessarily constitute or imply its endorsement, recommendation, or favoring by the United States government or Lawrence Livermore National Security, LLC. The views and opinions of authors expressed herein do not necessarily state or reflect those of the United States government or Lawrence Livermore National Security, LLC, and shall not be used for advertising or product endorsement purposes.

This work performed under the auspices of the U.S. Department of Energy by Lawrence Livermore National Laboratory under Contract DE-AC52-07NA27344. 


\title{
Image Reconstruction of Imatron Electron-Beam Tomography Data
}

\author{
Kyle Champley, Steve Azevedo, Dan Schneberk, Harry Martz \\ Lawrence Livermore National Laboratory \\ Livermore, CA 94551
}

\section{Executive Summary}

In this paper we describe the development of a filtered backprojection image reconstruction algorithm for an Imatron Electron-Beam Tomography (EBT) scanner. Raw data, software to perform data correction routines, and a technical document on the data format were all provided by Imatron. This document describes the basic geometry of the EBT scanner and the steps of the image reconstruction algorithm. Finally we demonstrate that the image quality our reconstruction algorithm is comparable with the reconstructed images provided by Imatron on an airport luggage data set.

\section{Introduction}

Electron-Beam Tomography (EBT) enables imaging of the linear attenuation coefficients of the object in the scanning field of view by measuring the attenuation of X-rays through the object. See Figure 1 for a sketch of an EBT scanner.

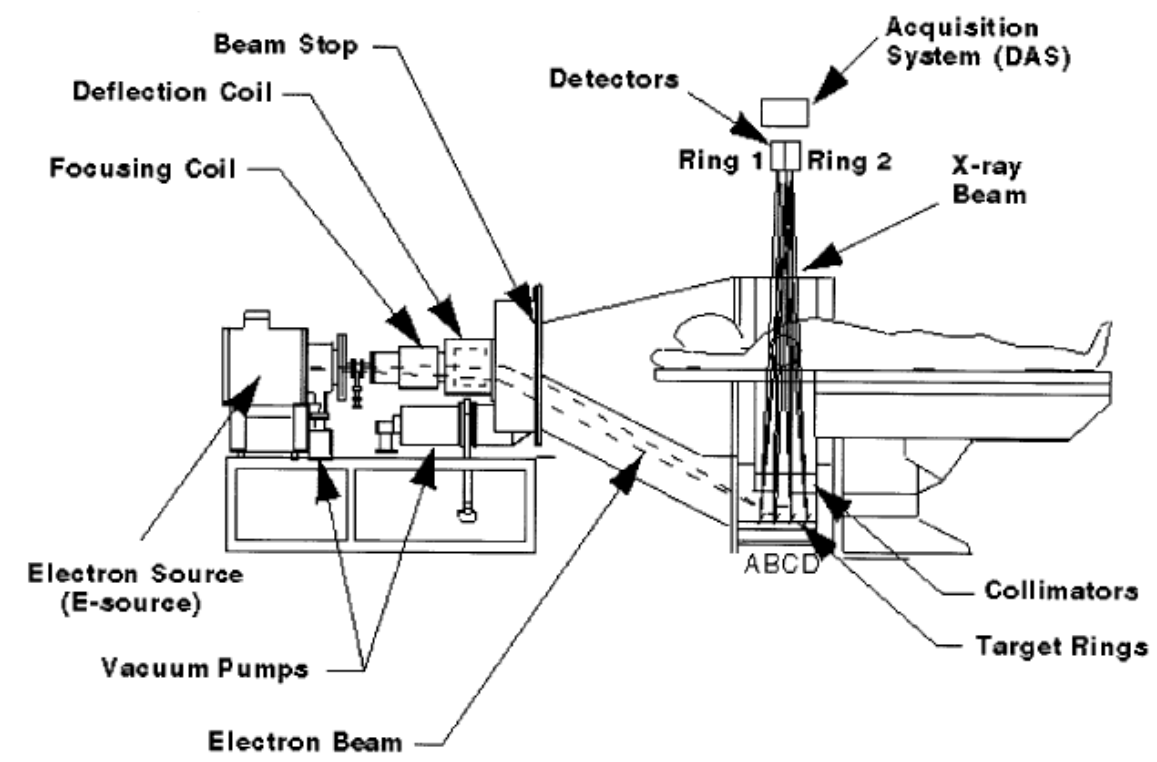

Figure 1: Schematic representation of an EBT Scanner 
We set out to reconstruct an image from an Imatron EBT scanner in helical mode. In the helical mode, the table upon which the object lies is continuously translated through the scanner while the circular projection data is being acquired. This mode enables threedimensional image reconstruction. Since the Imatron EBT scanner has only one detector row, we may reconstruct the helical data as a stack of two-dimensional images.

\section{Data Acquisition}

Now we focus our attention to the data format of the Imatron EBT scanner. This information was acquired through an internal Imatron technical document [1]. The X-ray detectors (864 of them) are arranged in a circular arc with a detector sampling of 0.25 degrees. The X-ray sources lie on a circular arc opposite the detectors. The detector arc and source arc share a common center point which we will refer to as the centered pin. The detectors are collimated with an acceptance angle of 41.267 degrees which constitutes $888 \mathrm{X}$-ray source locations. We will refer to the fan angle as the angle between the line from a fixed detector to the centered pin and the line from the detector to an X-ray source location. The fan angle will be denoted by $\alpha$. The azimuthal angle of a detector element will be denoted by $\beta$. The measured data are stored in an $864 \times 888$ matrix $(\beta \times \alpha)$. A sketch of these geometrical aspects of the scanner is shown in Figure 2.
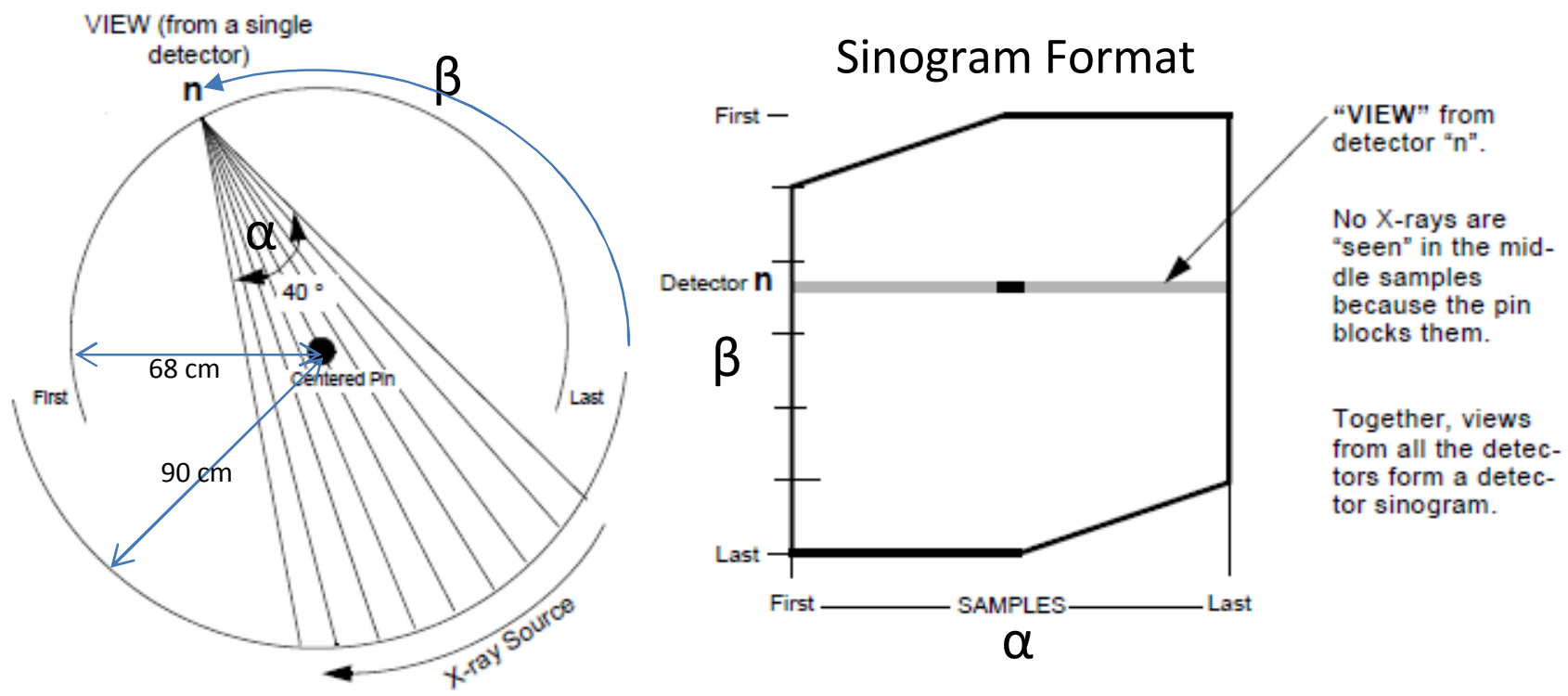

Figure 2: View from a Single Detector 
The raw data in this form, called a sinogram, must be corrected for a few effects (bad pixel correction, gain correction, missing views, etc) before the image can be reconstructed. Figure 3 shows the uncorrected (raw) and corrected sinogram data. The correction routines were provided by Imatron.

\section{Before Corrections}

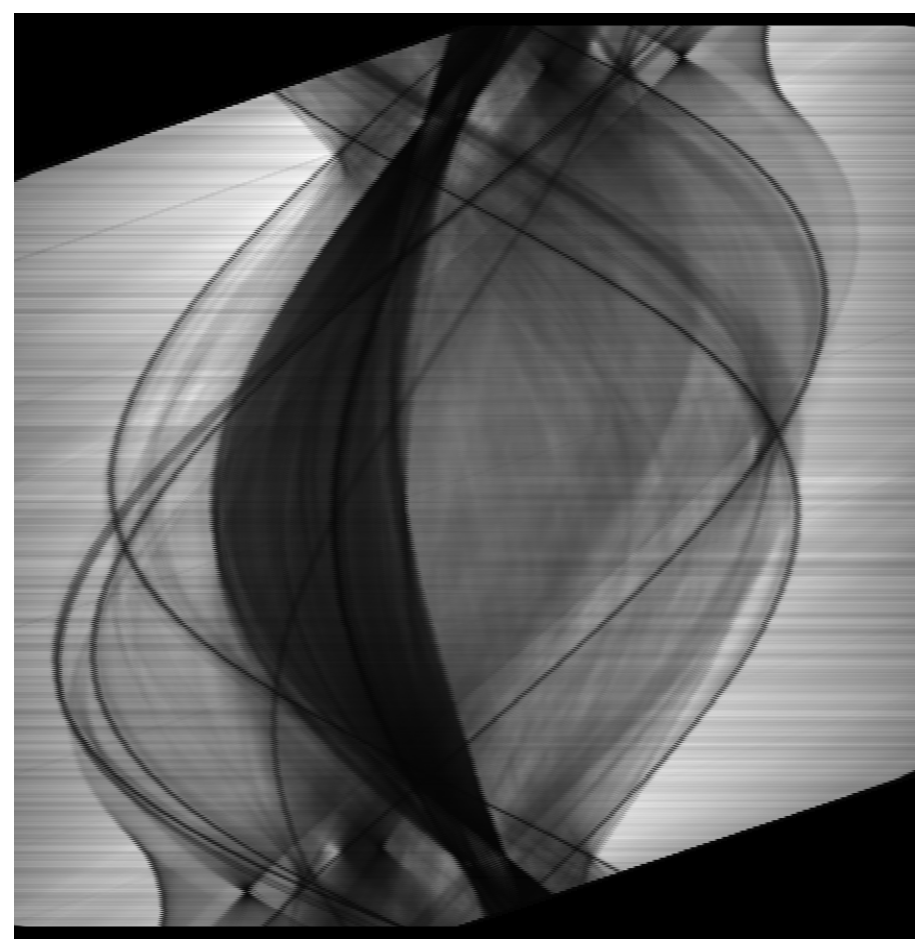

After Corrections

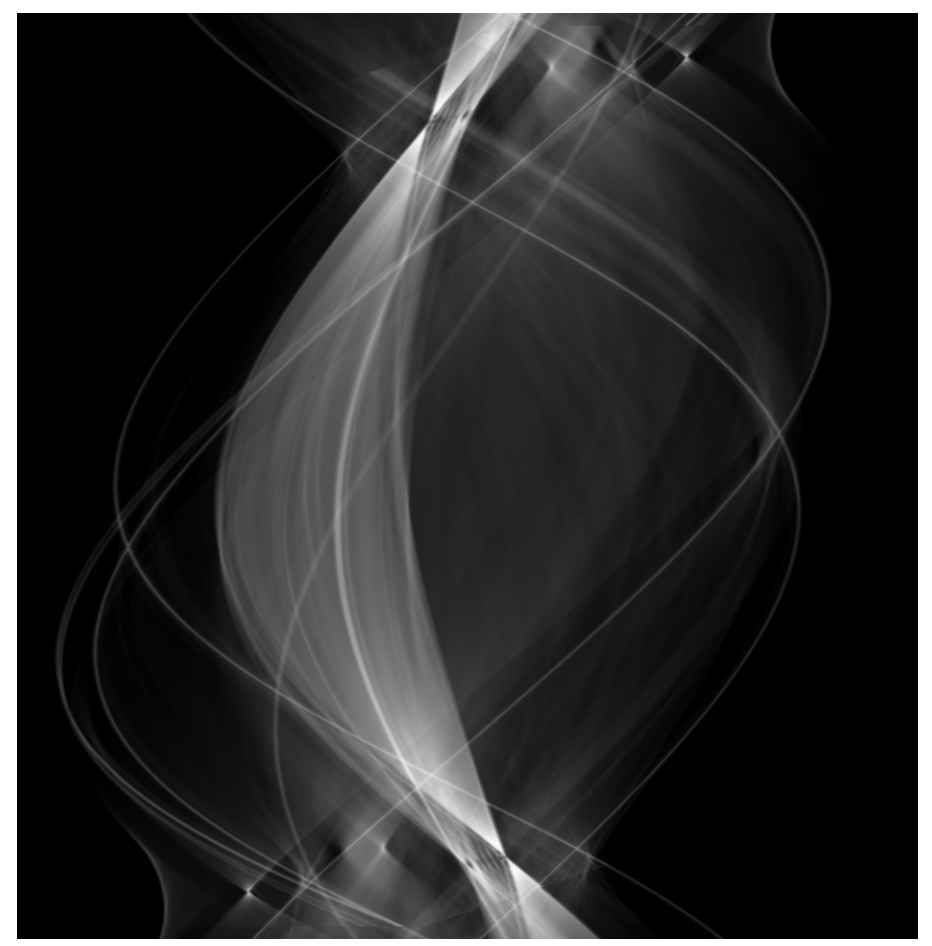

Figure 3: Uncorrected and Corrected Sinograms

\section{Data Reduction}

The image reconstruction process is implemented by filtered backprojection [2]. This method is performed in three steps. First, the data is rebinned using bilinear interpolation into so-called parallel beam coordinates denoted $(\mathrm{s}, \varphi)$, where

$$
\mathrm{s}=\operatorname{R} \sin (\alpha), \varphi=\beta+\alpha \text {, }
$$

and $\mathrm{R}=68 \mathrm{~cm}$ (detector-to-centered-pin distance). This transformation is visually displayed in Figure 4. Next, the data is filtered over parallel lines (over the s variable) with the Shepp-Logan ramp filter, which is given by

$$
h[n]=\frac{-1}{\pi\left(n^{2}-\frac{1}{4}\right)} \text {. }
$$


Finally, the filtered sinogram is backprojected. We employ the distance-driven backprojection algorithm, which is highly accurate and is well-suited for iterative reconstruction should we choose this option in the future.

Fan Beam Sinogram

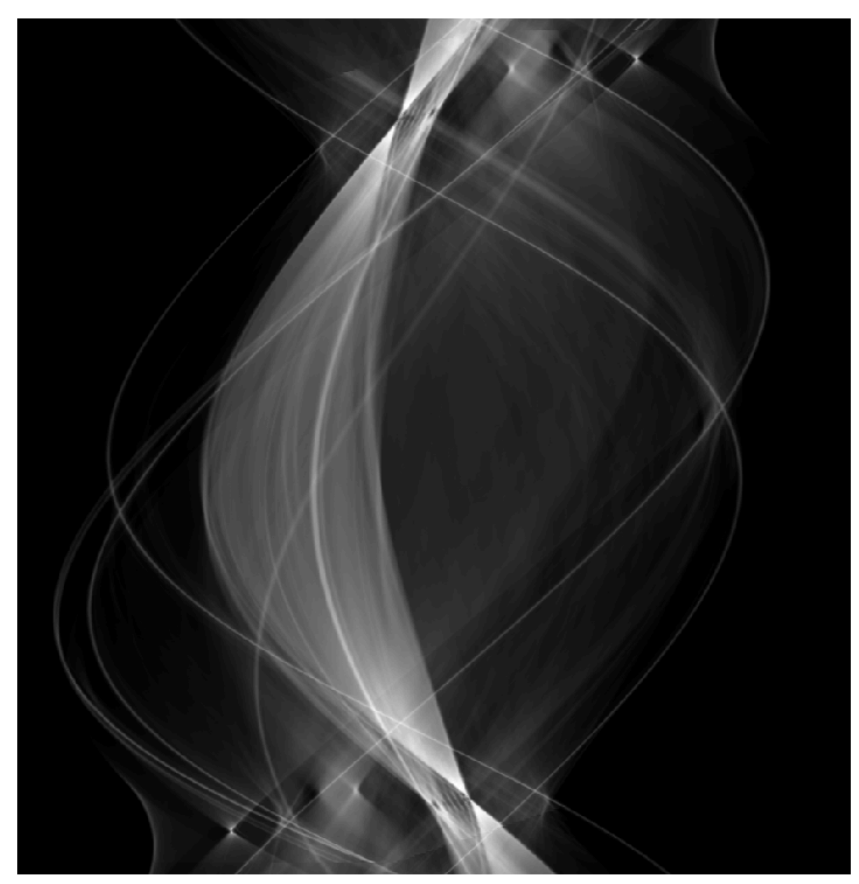

Figure 4: Fran Beam and Parallel Beam Sinograms
Parallel Beam Sinogram

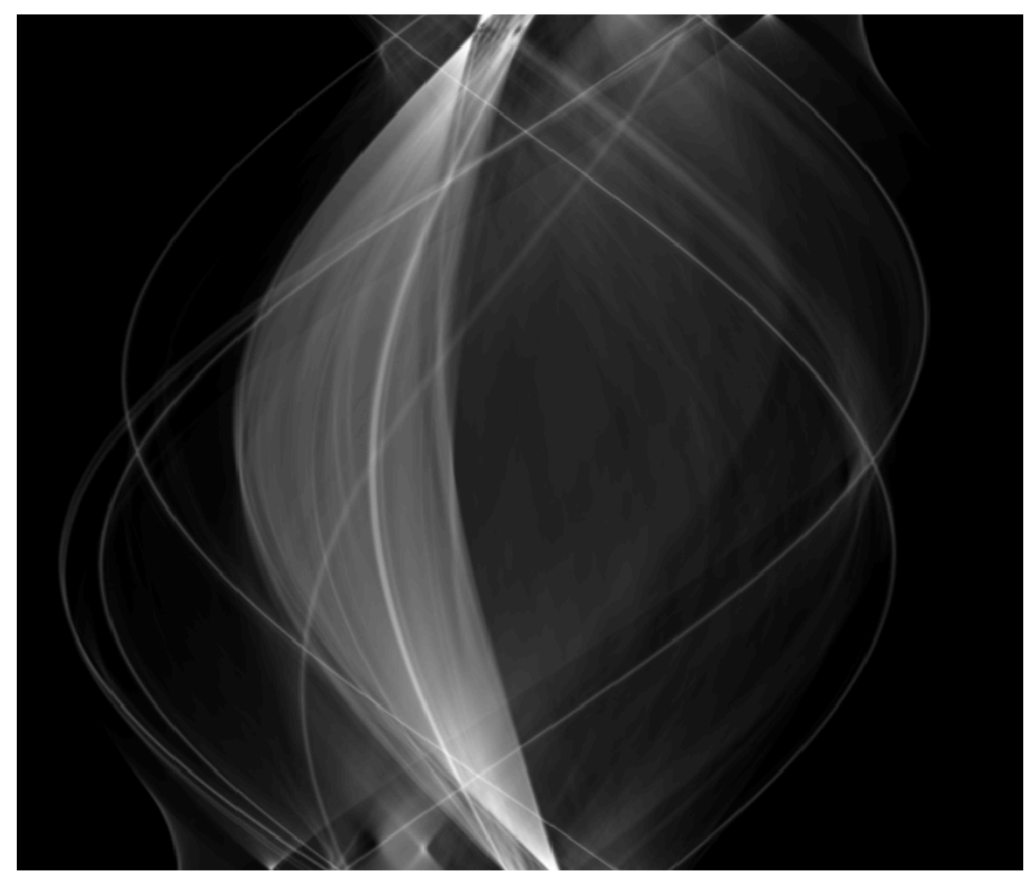

To verify our image reconstruction code we reconstructed data provided by Imatron and compared it to an Imatron reconstruction. The Imatron and LLNL reconstructed images are shown in Figure 5. There is an unknown scaling difference between the two images and the color scales were chosen, so the images look similar. However, there are some subtle differences and overall the Imatron image has better image quality, which is likely due to the fact that we used the approximate scanner geometry while Imatron used the exact geometry. For instance, the detectors are not located on a perfect circular arc as our image reconstruction algorithm assumes. The detector panel is actually composed of several detector modules, each containing a number of individual detectors and thus the detectors lie in a polygonal arrangement. 

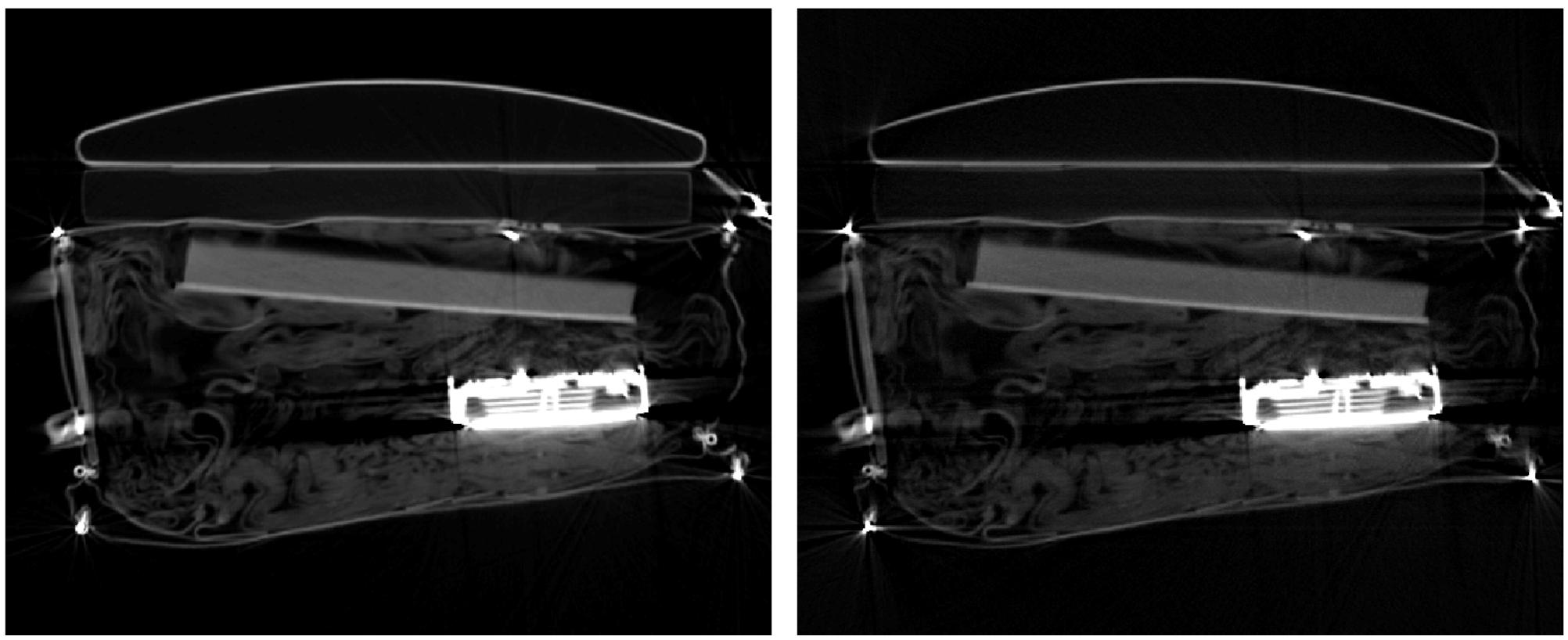

Figure 5: Reconstructed images. (left) Imatron Reconstruction. (right) LLNL Reconstruction.

\section{Summary}

This document has provided a detailed summary of the basic geometry of an Imatron EBT scanner and the steps needed to perform a filtered backprojection image reconstruction. The accuracy of our algorithm was demonstrated by showing that the image quality of our reconstruction algorithm is comparable to the reconstructed images provided by Imatron on an airport luggage data set.

\section{Future Work}

To improve the image quality of the reconstruction one should obtain the exact scanning geometry from Imatron and incorporate it into the reconstruction algorithm.

\section{References}

[1] Imatron C300 Technical Support Manual, Vol 13: Sinograms, 2007

[2] Adel Faridani, Introduction to the Mathematics of Computed Tomography, Inside Out: Inverse Problems, MSRI Publications, Volume 47, 2003 